Disclosure of Interests: Sophie De Mits: None declared, Liselotte Deroo: None declared, Ann-Sophie De Craemer: None declared, Thomas Renson: None declared, Tine Willems: None declared, Lieven Danneels: None declared, Philippe Carron: None declared, Filip van den Bosch Consultant for: AbbVie, BMS, Galapagos, Janssen, Lilly, Merck, Novartis, Pfizer and UCB, Speakers bureau: AbbVie, BMS, Janssen, Lilly, Merck, Novartis, Pfizer and UCB., Dirk Elewaut: None declared

DOI: 10.1136/annrheumdis-2019-eular.6868

\section{AB1386-HPR FORCE SENSE EVALUATION OF THE KNEE JOINT FOLLOWING MENISCECTOMY RELATED WITH DEGENERATIVE MENISCAL TEAR}

Cansu Gevrek ${ }^{1}$, Gizem Irem Kinikli', Ahmet Ozgur Atay ${ }^{2} .{ }^{1}$ Hacettepe University, Faculty of Physical Therapy and Rehabilitation, Ankara, Turkey; ${ }^{2}$ Hacettepe University Faculty of Medicine, Department of Orthopaedics and Traumatology, Ankara, Turkey

Background: Meniscal tears are associated with degenerative knee disease, which can range from mild chondral changes not visible on a radiograph to established knee osteoarthritis. Meniscectomy related with degenerative meniscal tear may affect the force sense of the knee joint which is defined as the ability to accurately reproduce a given force.

Objectives: The aim of this study was to compare the force sense of the knee joint of the patients with degenerative meniscal tear following meniscectomy with healthy peers.

Methods: Fifteen patients with meniscectomy and 18 healthy aged matched individuals were included in this study. Evaluation of force sense in the knee joint was measured with the use of a pressure biofeedback device, sphygmomanometer, (Stabilizer, TM, Chattanooga Group Inc., Chattanooga, TM). The pressure bag of this device was placed under the knee joint. All subjects were instructed to extend the knee via Maximal Voluntary Isometric Contraction (MVIC) for $5 \mathrm{~s}$. Each measurement was repeated three times with 1 -min rest intervals and the average constant errors observed on the pressure biofeedback device were recorded as $\mathrm{mmHg}$. Subjects were instructed to obtain at $50 \%$ of MVIC. They were asked to maintain the contraction (reproduce the target force) for $5 \mathrm{~s}$. The reproduction force was subtracted from the target force to create a trial error score. Higher error scores indicated lower force sense. Mann Whitney $U$ test was used for group comparisons. Statistical significance was defined as a $p$ value $<0.05$.

Results: There were no difference in terms of mean age, body mass index between patients $\left(53.67 .2\right.$ years, $\left.26.21 .8 \mathrm{~kg} / \mathrm{m}^{2}\right)$ and healthy peers $\left(53.47 .09\right.$ years, $\left.25.83 .2 \mathrm{~kg} / \mathrm{m}^{2}\right)$, respectively $(p>0.05)$. Lower accuracy of the knee extensors during the $50 \%$ MVIC task was significantly seen in patients with meniscectomy $(5.422 .07 \mathrm{mmHg})$ compared to healthy group $(1.881 .1 \mathrm{mmHg})(p<0.001)$

Conclusion: Results of this study demonstrated that higher force sense deficits are present in patients with meniscectomy related to degenerative meniscal tear compared to healthy peers. Thus, knee proprioceptive training focus on force sense might be developed to improve functional capabilities of knee joint especially following meniscectomy. It is also important to establish effective and feasible evaluation interventions for health professionals in clinical practice for assessing proprioception in terms of force sense in degenerative knees.

\section{REFERENCES}

[1] Sihvonen, R., Paavolah, M,D., Malmivaara, A., Itl, A., Joukainen, A., Nurmi, H., Halske, J., Jrvinen T.L.N. (2013). Arthroscopic Partial Meniscectomy versus Sham Surgery for a Degenerative Meniscal Tear. The new england journal of medicine, 369:2515-2524.

[2] Jarraya, M., Roemer, F.W., Englund, M., Crema, M.D., Gale, H.I., Hayashi, D., Katz J,N,. Guermazi A. (2017). Meniscus morphology: Does tear type matter? A narrative review with focus on relevance for osteoarthritis research. Seminars in Arthritis and Rheumatism 46:552561.

Disclosure of Interests: None declared

DOI: 10.1136/annrheumdis-2019-eular.3831

\section{AB1387-HPR ASPECTS OF VALIDITY AND RELIABILITY OF THE SWEDISH VERSION OF PATIENT HEALTH QUESTIONNAIRE-8 IN INDIVIDUALS WITH SYSTEMIC SCLEROSIS}

Malin Mattsson ${ }^{1}$, Gunnel Sandqvist ${ }^{2}$, Roger Hesselstrand ${ }^{2}$, Elisabet Dyrsmeds ${ }^{3}$, Annika Nordin ${ }^{4}$, Carina Boström ${ }^{1} .{ }^{1}$ Karolinska Institutet, Department of Neurobiology, Care sciences and Society, Stockholm, Sweden; ${ }^{2}$ Lund University, Department of Clinical Sciences, Lund, Sweden; ${ }^{3}$ Karolinska University Hospital, Unit of Rheumatology, Stockholm, Sweden; ${ }^{4}$ Karolinska Institutet, Department of Medicine, Stockholm, Sweden

Background: For assessing symptoms of depression in systemic sclerosis (SSc), the Patient Health Questionnaire-8 (PHQ-8) may be useful in clinical care as it is short and easy to administer. The English version of PHQ-8 has been found to be reliable and valid in SSc.

Objectives: To assess aspects of validity and reliability of PHQ-8 in Swedish (PHQ-8 Swe) for individuals with SSc.

Methods: Patients meeting the 2013 ACR/EULAR SSc criteria were recruited. The PHQ-8 Swe content validity was assessed via individual interviews (11 patients, 10 healthcare professionals, HPs) which was transcribed and analysed by content analysis. Patients ages, disease durations and symptoms of depression varied. The HPs had different occupational backgrounds and experiences in SSc care. Reliability was tested by internal consistency and test-retest reliability. Sixty-seven patients (median age 62 [minmax: 2887]) completed the PHQ-8 Swe on two different occasions.

Results: Content validity: The instruction, items, and response options were generally considered easy to understand; however, some clarifications were suggested. Among HPs, it was expressed that some items could be perceived as emotionally demanding for patients especially in recent onset disease. Further they experienced that PHQ-8 was problemrather than possibility-based. Introducing the PHQ-8 to patients and the need for follow-ups of the answers to the questionnaire were stated as essential. It was further expressed that PHQ-8 covered key aspects of symptoms of depression in SSc nevertheless examples of items suggested to be included were purpose in life, thoughts about death, and loneliness. The items were overall experienced as important without redundancy, and that some items could reflect more general SSc-related symptoms.

Cultural adaption: Possible adjustments to the PHQ-8 translation were made. This version was back translated into English and compared with the original English version. Thereafter, minor changes were made. A final PHQ-8 Swe version was tested for reliability.

Reliability: Cronbachs alpha was 0.86 and the corrected item-to-total correlation range was 0.420 .78 . There were no significant differences between testretest for seven of the eight items. The median weighted kappa coefficient was 0.63 . The median PHQ-8 Swe total score was 4 (020, no-severe depressive symptoms). There were no significant differences for total scores between test and retest and the ICC was 0.81 .

Conclusion: Content validity of PHQ-8 Swe was satisfactory and a positive quality was indicated for aspects of reliability in individuals with SSC tested in this study. Although some items could be interpreted as covering more general SSc-related symptoms, the PHQ-8 could be valuable for detecting patients with symptoms of depression in need of discussing emotional issues, as reflected in our study. Further studies of the PHQ-8 Swes usefulness are currently being undertaken via evaluation of other psychometric aspects.

\section{REFERENCES}

[1] -Kroenke K, et al. The PHQ-8 as a measure of current depression in the general population. J Affect Disord. 2009; 114:163-73

[2] -Milette K, et al. Comparison of the PHQ-9 and CES-D depression scales in systemic sclerosis: internal consistency reliability, convergent validity and clinical correlates. Rheumatology. 2010; 49:789-96

\section{ACKNOWLEDGEMENT}

Approval to adjust a translation of PHQ-8 to Swedish for patients with SSc was received from one of the original developers. We thank patient research partners Monica Holmnr and Astrid von Rosen for their valuable contribution. Funding was received from Region Norrbotten.

Disclosure of Interests: None declared

DOI: 10.1136/annrheumdis-2019-eular.4076 